\title{
Factors Associated With Pediatric Emergency Airway Management by the Difficult Airway Response Team
}

Nicholas M. Dalesio ${ }^{1,} 2$, Lauren Burgunder ${ }^{3}$, Natalia M. Diaz-Rodriguez ${ }^{2}$, Sara I. Jones ${ }^{2}$, Jordan DuvalArnould $^{4}$, Laeben C. Lester ${ }^{2}$, David E. Tunkel ${ }^{1}$, Sapna R. Kudchadkar ${ }^{2}$

1. Otolaryngology/Head \& Neck Surgery, Johns Hopkins University School of Medicine, Baltimore, USA 2. Anesthesiology and Critical Care, Johns Hopkins University School of Medicine, Baltimore, USA 3. Pediatrics, Johns Hopkins University School of Medicine, Baltimore, USA 4. Johns Hopkins Medicine Simulation Center, Johns Hopkins University School of Medicine, Baltimore, USA

Corresponding author: Nicholas M. Dalesio, ndalesi1@jhmi.edu

\section{Abstract}

\section{Background}

The goal of this study was to determine if difficult airway risk factors were similar in children cared for by the difficult airway response team (DART) and those cared for by the rapid response team (RRT).

\section{Methods}

In this retrospective database analysis of prospectively collected data, we analyzed patient demographics, comorbidities, history of difficult intubation, and intubation event details, including time and place of the emergency and devices used to successfully secure the airway.

\section{Results}

Within the 110-patient cohort, median age (IQR) was higher among DART patients than among RRT patients [8.5 years $(0.9-14.6)$ versus 0.3 years $(0.04-3.6) ; \mathrm{P}<0.001$ ]. The odds of DART management were higher for children ages $1-2$ years (aOR, 43.3; 95\% CI: 2.73-684.3) and $>5$ years (aOR, 13.1; 95\% CI: $1.85-93.4$ ) than for those less than one-year-old. DART patients were more likely to have craniofacial abnormalities (aOR, 51.6; 95\% CI: 2.50-1065.1), airway swelling (aOR, 240.1; 95\% CI: 13.6-4237.2), or trauma (all DART managed). Among patients intubated by the DART, children with a history of difficult airway were more likely to have musculoskeletal $(\mathrm{P}=0.04)$ and craniofacial abnormalities $(\mathrm{P}<0.001)$, whereas children without a known history of difficult airway were more likely to have airway swelling $(\mathrm{P}=0.04)$.

\section{Conclusion}

Review began 04/26/2021 Review ended 05/03/2021 Published 07/02/2021

(c) Copyright 2021 Dalesio et al. This is an open access article distributed under the terms of the Creative Commons Attribution License CC-BY 4.0., which permits unrestricted use, distribution, and reproduction in any medium, provided the original author and source are credited.
Specific clinical risk factors predict the need for emergency airway management by the DART in the pediatric hospital setting. The coordinated use of a DART to respond to difficult airway emergencies may limit attempts at endotracheal tube placement and mitigate morbidity.

Categories: Anesthesiology, Pediatrics

Keywords: airway disorders, difficult airway management, pediatric anesthesiology, interests in difficult airway and regional anaesthesia, multi-disciplinary teams, rapid response teams

\section{Introduction}

Difficult airways in children are rare; however, airway-related complications are a significant cause of morbidity and mortality. In the pediatric operating suite (OR), approximately $0.02 \%$ of cases involve difficult mask ventilation and $0.25 \%$ to $0.32 \%$ of cases have difficult intubation [1,2]. A 2014 publication reported that outside the OR, in the pediatric intensive care unit (PICU), approximately $9 \%$ of all tracheal intubations could be classified as difficult, requiring three or more attempts [3]. Senior-level practitioners performed most of the initial intubation attempts in such cases (81\%), yet severe adverse events, including cardiac arrest, esophageal intubation with delayed recognition, and emesis with witnessed aspiration, remained high, at $13 \%$.

Both the Joint Commission on Accreditation of Healthcare Organizations and the Institute for Healthcare Improvement via the 100,000 Lives Campaign recommend that hospitals have "a system of rapid response teams (RRTs) to bring skilled resources” [4]. Most hospitals have RRTs for first-line assessment and management of patients with acute clinical deterioration. At our institution, the pediatric RRT consists of an intensivist, a PICU fellow, a respiratory therapist, and a pediatric nurse, and intubation is performed by the in-house PICU fellow or attending. However, a team of airway experts can provide critical support and personnel proficient in advanced airway management devices and techniques not routinely used outside the 
OR. Therefore, in 2008, our institution created the difficult airway response team (DART) composed of anesthesiologists, intensivists, nurses, respiratory therapists, otolaryngology-head and neck surgeons, and trauma surgeons, to manage adults and children with a difficult airway in inpatient settings [5]. The DART team is routinely called if (1) a patient with history of difficult airway is in respiratory distress, (2) multiple attempts at securing the airway have failed, or (3) difficulty securing the airway is likely, based on the mechanisms for respiratory compromise.

Difficulties in pediatric airway management can often be predicted by the presence of specific risk factors identified via medical history and physical examination [6,7]. However, specific patient and contextual factors (e.g., time of day, location) associated with airway management by the DART have not been described in the pediatric inpatient setting. Thus, we sought to compare demographic, clinical, and situational risk factors of pediatric inpatients who were successfully intubated by the RRT to those who required the DART at our tertiary-care hospital.

\section{Materials And Methods}

The institutional review board (IRB) at our university approved the study protocol (IRB\# NA_00089582) and waived the requirement for informed consent.

\section{Study design}

We conducted a retrospective database analysis of prospectively collected data from the DART registry between February 1, 2009, and December 31, 2015. The DART registry is a prospectively collected database of all DART calls in which the responding provider enters demographic data and details regarding airway management during the DART response. Patients were included if they were less than 15 years of age or were cared for in the children's center. We compared characteristics of children intubated by the DART to those of children in the Resuscitation Event Analysis Clearinghouse (REACH) Surveillance System [8] who were intubated by the RRT between January 24, 2013, and March 14, 2016. If the RRT had difficulty securing the airway of a patient, and consequently activated the DART for intervention, we included that patient in the DART dataset (Figure 1). Patients with a known history of difficult airway had initial airway management performed by the DART.

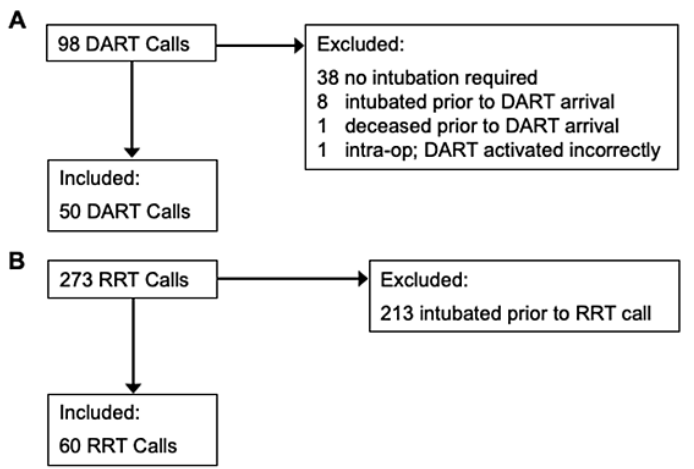

\section{FIGURE 1: Flow chart for inclusion and exclusion of (A) difficult airway} response team (DART) and (B) rapid response team (RRT) cases.

We collected demographic data including patient age, weight, history of difficult airway, and comorbid conditions. Information about airway pathology, including airway bleeding and trauma, was also collected. Intubation devices and attempts, patient location (e.g., inpatient floor, emergency department [ED], PICU, and neonatal intensive care unit [NICU]), whether cardiopulmonary resuscitation (CPR) was underway during intubation, and provider training level were also noted. In several situations, efforts were made to mobilize pediatric DART patients to the OR for use of advanced airway management techniques and devices. 


\section{Cureus}

\section{Statistical analysis}

Categorical data were analyzed by chi-squared test, and continuous data (expressed as medians with interquartile range $[\mathrm{IQR}]$ ) were analyzed by the Mann-Whitney U-test. We applied univariate analysis as well as multivariable logistic regression modeling to determine the differences in patient comorbidities and intubation scenarios between the groups. A P-value $<0.05$ was considered statistically significant. Stata SE 15.1 (StataCorp, College Station, TX) was used to conduct the statistical analysis.

\section{Results}

\section{Demographics}

A total of 110 patients ( $45 \%$ female) were eligible for inclusion in the analysis. Sixty patients were intubated by the RRT (54.5\%), and the remaining 50 patients had intervention by the DART. In the latter group, 29 (58\%) had a history of difficult airway, and 21 (42\%) had unanticipated difficult airway. Three patients had more than two DART calls, either during one or multiple hospitalizations. As shown in Table 1, children in the RRT group were significantly younger (median age 0.3 years [IQR, 0.04-3.6]) than children intubated by the DART (median age 8.5 years [IQR, 0.9-14.6]; $\mathrm{P}<0.001$ ).

\begin{tabular}{|c|c|c|c|c|}
\hline Characteristic & Total $(n=110)$ & RRT intubation $(n=60)$ & DART intubation $(n=50)$ & P-value ${ }^{a}$ \\
\hline Age, years, median (IQR) & $1.1(0.1-10.6)$ & $0.3(0.04-3.6)$ & $8.5(0.9-14.6)$ & $<0.001$ \\
\hline Age category, n (\%) & & & & $<0.001$ \\
\hline$<1$ year & $51(46)$ & $38(63)$ & $13(26)$ & \\
\hline $1-2$ years & $11(10)$ & $3(5)$ & $8(16)$ & \\
\hline $2-5$ years & $12(11)$ & $9(15)$ & $3(6)$ & \\
\hline$>5$ years & $36(33)$ & $10(17)$ & $26(52)$ & \\
\hline Sex, n (\%) & & & & 0.15 \\
\hline Male & $60(55)$ & $29(48)$ & $31(62)$ & \\
\hline Female & $50(45)$ & $31(52)$ & $19(38)$ & \\
\hline Weight, kg, median (IQR) ${ }^{\mathrm{b}}$ & $(3.2-29.7)$ & $4.2(1.7-12.9)$ & $18.4(7.5-57)$ & $<0.001$ \\
\hline
\end{tabular}

TABLE 1: Demographic data of patients intubated by the rapid response team and those intubated by the difficult airway response team.

DART: difficult airway response team; IQR: interquartile range; RRT: rapid response team.

aStatistical differences were assessed with the Mann-Whitney test for nonparametric continuous data and with the Pearson's chi-squared test for categorical variables.

${ }^{b}$ Weight was missing for one patient in the RRT group.

\section{Location of airway management}

The most common location for intubations by both RRT (65\%) and DART (70\%) were the intensive care units (ICUs). RRTs occurred more commonly in the NICU, whereas DARTs occurred more commonly in the PICU (RRT: $42 \%$ NICU, 23\% PICU; DART: $8 \%$ NICU, 62\% PICU). RRT and DART had similar percentages of response calls to the ED ( $18 \%$ and $22 \%$, respectively, $\mathrm{P}=0.54)$. Both RRT- and DART-led intubations took place in the OR only when patients were transferred there emergently from another location for use of advanced airway management techniques and equipment stored there. Patients located on the inpatient floor were more likely to be successfully cared for by the RRT and not require the DART ( $n=5$ RRT; $n=1$ DART; Table 2).

\begin{tabular}{|c|c|c|c|c|}
\hline Characteristic & Total $(n=110)$ & RRT intubation $(n=60)$ & DART intubation $(n=50)$ & P-value ${ }^{a}$ \\
\hline Intubation location, n (\%) & & & & 0.54 \\
\hline ICU & $73(66)$ & $39(65)$ & $35(62)$ & \\
\hline
\end{tabular}




\section{Cureus}

\begin{tabular}{|c|c|c|c|c|}
\hline Medical floor & $6(6)$ & $5(8)$ & $1(2)$ & \\
\hline Operating suite ${ }^{\mathrm{b}}$ & 6(5) & $4(7)$ & $3(6)$ & \\
\hline PACU & 1 (1) & 1(2) & 0 & \\
\hline Emergency department & $24(22)$ & $11(18)$ & $11(22)$ & \\
\hline Time of day, $n(\%)^{c}$ & 102 & 60 (59) & $42(41)$ & 0.04 \\
\hline $6 \mathrm{am}-12 \mathrm{pm}$ & $38(37)$ & $14(23)$ & $24(57)$ & \\
\hline $12 \mathrm{pm}-6 \mathrm{pm}$ & $20(20)$ & $15(25)$ & $4(10)$ & \\
\hline $6 \mathrm{pm}-12 \mathrm{am}$ & $16(16)$ & $16(27)$ & 1(2) & \\
\hline 12 am-6 am & $28(27)$ & $15(25)$ & $13(31)$ & \\
\hline \multicolumn{5}{|l|}{ Intubation attempts ${ }^{d}$} \\
\hline Median (IIR) & $1(1-3)$ & $1(1-5)$ & $2.5(1-4)$ & $<0.001$ \\
\hline Before DART calle & & NA & $0(0-1.5)$ & \\
\hline After DART call & & NA & $1(1-2.5)$ & \\
\hline Rank of successful clinician, $n(\%)$ & & & & 0.25 \\
\hline Attending & 29 & $13(22)$ & $16(32)$ & \\
\hline Fellow & 19 & $13(22)$ & $6(12)$ & \\
\hline Resident or nurse practitioner & 3 & $2(3)$ & $1(2)$ & \\
\hline Not recorded & 59 & $32(53)$ & $27(54)$ & \\
\hline Intubation techniques attempted & & & & $<0.001$ \\
\hline Direct laryngoscopy with RSI & 64 & 59 & 5 & \\
\hline Direct laryngoscopy without RSI & 9 & 0 & 9 & \\
\hline Videolaryngoscopy (GlideScope® or CMAC®) & 7 & 1 & 6 & \\
\hline Fiberoptic intubation & 6 & 0 & 6 & \\
\hline Fiberoptic intubation via SGA & 5 & 0 & 5 & \\
\hline Rigid laryngoscopy ${ }^{f}$ & 12 & 0 & 12 & \\
\hline Surgical aimway ${ }^{9}$ & 7 & 0 & 7 & \\
\hline
\end{tabular}

TABLE 2: Comparison of patient groups that underwent invasive airway management by the rapid response team (RRT) and by the difficult airway response team (DART).

DART: difficult airway response team; ICU: intensive care unit; NA: not applicable; PACU: post-anesthesia care unit; CPR: cardiopulmonary resuscitation; RRT: rapid response team; RSI: rapid sequence intubation; SGA: supraglottic airway.

aStatistical differences were assessed by the Mann-Whitney test for nonparametric data for continuous variables and Pearson's chi-squared test for categorical variables.

${ }^{b}$ Efforts are made to move patients to the operating room suite if advanced airway techniques are needed (e.g., fiberoptic bronchoscopes, inhalational anesthesia). DART calls are not made on patients already in the operating suite.

${ }^{\mathrm{C}}$ Eight DART patients did not have a time of day registered.

dOne patient had care withdrawn after intubation attempts.

${ }^{\text {e} A t t e m p t s ~ b e f o r e ~ D A R T ~ a c t i v a t i o n ~ w e r e ~ n o t ~ r e c o r d e d ~ f o r ~ t w o ~ p a t i e n t s . ~ A l l ~ p a t i e n t s ~ w i t h ~ h i s t o r y ~ o f ~ d i f f i c u l t ~ a i r w a y ~ h a d ~ i n i t i a l ~ i n t u b a t i o n ~ a t t e m p t s ~}$ performed by the DART.

${ }^{f}$ Rigid laryngoscopy is defined as intubation by the otolaryngologist using operative laryngoscopes or a rigid bronchoscope. 


\section{Time of day for RRT and DART activation}

Over half of DART calls with a known time of day (24 of 42) occurred between 6 am and 12 pm, compared with only $23 \%$ ( 14 of 60 ) of RRT calls $(\mathrm{P}=0.04)$. The second most common time for DART activation was during late-night hours between 12 am and 6 am (31\%; Table 2).

\section{Intubation attempts}

Of the 60 RRT patients, 44 (73\%) were intubated after one attempt and 52 (87\%) had a secure airway within three attempts. Eight RRT patients (13\%) required more than three attempts to secure the airway, of which, five were neonates under 1 month of age and one had a craniofacial abnormality. One patient required up to six attempts (Table 2).

The median number of attempts to secure the airway in DART patients was 2.5 (IQR, 1-4), and 74\% had their airway secured within three attempts. In DART activations where the difficult airway was not anticipated, the ED or ICU staff performed between three and six intubation attempts; however, intubation attempts prior to DART activation were not documented in two of the 50 patients. Three patients required six attempts and one patient required 10 attempts (six attempts prior to DART activation and four attempts by the DART) to secure the airway. After the DART responded, 27 of 48 (56.3\%) were intubated on the first attempt, and 44 of 48 (91.7\%) were intubated in three or fewer attempts (Table 2). One DART patient with an unanticipated difficult airway died during intubation in a "cannot ventilate, cannot intubate" scenario. No patients died during intubation by the RRT.

The physician's experience and seniority of the clinician who secured the airway were not consistently recorded and were missing in 32 of the 60 (53.3\%) RRT records and 27 of the 50 (54.0\%) DART records. Among complete records, the attending or fellow successfully intubated 26 of the 28 (92.9\%) patients in the RRT group and 22 of the 23 (95.7\%) patients in the DART group (Table 2).

\section{Intubation techniques}

Almost all of the RRT intubations were performed by direct laryngoscopy with a rapid sequence induction ( 59 of 60). One patient in the ICU who required intubation because of blood in the airway was intubated after one attempt by the RRT using a videolaryngoscope. Thirty-six of the 50 DART patients (72.0\%) required an advanced technique for intubation. Twelve (33.3\%) of those who required advanced techniques were successfully intubated by an otolaryngologist using operative laryngoscopes with or without a rigid bronchoscope, and 11 (30.6\%) required fiberoptic intubation either transorally or via a supraglottic airway. Six surgical airways were performed. One was a new tracheostomy in an 18-year-old who had asthma and subglottic stenosis likely caused by prolonged intubation. A 14-year-old patient received a needle cricothyrotomy, and four patients received revision tracheostomies (Table 2).

\section{Risk factors}

Most DART patients (84\%) had at least one difficult airway risk factor (Table 3) compared to only $27 \%$ (16 of 60 ) of RRT patients. In unadjusted analysis, the presence of one or more clinical difficult airway risk factors, such as bleeding, trauma, edema, difficult airway history, or musculoskeletal and craniofacial abnormality was highly significantly associated with intubation by the DART (odds ratio, 14.4; 95\% CI: 5.6-37.3). It was less common for patients undergoing CPR to require the DART for intubation (OR, 0.09; 95\% CI: 0.040.22 ; Table 3) 


\section{Cureus}

\begin{tabular}{|c|c|c|c|c|c|}
\hline Comorbiditya & Total & Crude OR $(95 \% \text { CI) })^{\mathrm{b}}$ & P-value ${ }^{c}$ & aOR $(95 \% \mathrm{Cl})^{\mathrm{d}}$ & P-value $^{c}$ \\
\hline \multicolumn{6}{|l|}{ Age } \\
\hline$<1$ year & 51 & 1 (reference) & $\cdot$ & 1 (reference) & \\
\hline 1-2 years & 11 & $7.8(1.79-33.9)$ & 0.01 & $43.3(2.73-684.3)$ & 0.007 \\
\hline 2-5 years & 12 & $0.97(0.23-4.16)$ & 0.97 & $2.2(0.09-54.1)$ & 0.63 \\
\hline$>5$ years & 36 & $7.6(2.90-19.9)$ & $<0.001$ & 13.1 (1.85-93.4) & 0.010 \\
\hline CPR during intubation & 56 & $0.09(0.04-0.22)$ & $<0.001$ & $0.04(0.01-0.23)$ & $<0.001$ \\
\hline Difficult airway historye & 29 & - & - & - & - \\
\hline Airway bleeding & 14 & $3.5(1.0-12.0)$ & 0.04 & $6.1(0.50-75.2)$ & 0.16 \\
\hline Airway swelling & 18 & $13.7(2.9-126.7)$ & $<0.001$ & 240.1 (13.6-4237.2) & $<0.001$ \\
\hline Trauma $^{e}$ & 16 & - & $\cdot$ & - & - \\
\hline Genetic syndromes & 13 & $0.49(0.14-1.71)$ & 0.27 & $1.5(0.15-15.9)$ & 0.72 \\
\hline Craniofacial diseases $^{t}$ & 15 & $10.2(2.2-47.8)$ & 0.003 & $51.6(2.50-1065.1)$ & 0.01 \\
\hline Musculoskeletal diseases & 11 & $14.8(1.8-119.8)$ & 0.01 & $4.2(0.12-145.1)$ & 0.43 \\
\hline \multicolumn{6}{|l|}{ Multiple risk factors } \\
\hline No risk factors & 52 & 1 (reference) & $\cdot$ & $\mathrm{NA}$ & \\
\hline One risk factor & 35 & $8.25(3.0-22.7)$ & $<0.001$ & NA & \\
\hline Two risk factors & 19 & $46.8(9.0-0-242.8)$ & $<0.001$ & NA & \\
\hline More than two risk factors ${ }^{e}$ & ${ }^{4}$ & $\cdot$ & $\cdot$ & & \\
\hline
\end{tabular}

TABLE 3: Univariate and multivariable adjusted odds ratios for comorbidities comparing patients with difficult intubation performed by the Difficult Airway Response Team and those undergoing intubation by the rapid response team

aOR: adjusted odds ratio; $\mathrm{Cl}$ : confidence interval; CPR: cardiopulmonary resuscitation; DART: difficult airway response team; NA: not applicable; OR: odds ratio.

${ }^{a}$ Difficult airway history indicates patients who had been diagnosed by an anesthesiologist in the past. Airway bleeding includes patients with posttonsillectomy bleeding and airway trauma. Airway swelling includes patients with anaphylaxis, airway burns or inhalational injury, subglottic stenosis, and airway trauma. Trauma includes patients who had been in a motor vehicle crash, had attempted suicide, or had a gunshot wound. Genetic syndromes include Algille's, Epidermolysis bullosa, and Moya Moya disease. Craniofacial diseases include Treacher-Collins, Pierre-Robin, Noonan's, and Hunter's syndrome. Musculoskeletal diseases include muscular dystrophy.

${ }^{\mathrm{b}}$ Crude estimates without adjustment for any covariate.

'Statistical differences were assessed with multivariable logistic regression modeling.

"Adjusted estimate includes all variables in the table except for the subgroups "Multiple risk factors" and "History of difficult airway" because all of these patients automatically have the DART called for any respiratory emergency.

${ }^{\text {e} A l l ~ p a t i e n t s ~ w e r e ~ c a r e d ~ f o r ~ b y ~ t h e ~ D A R T . ~}$

${ }^{f}$ Craniofacial conditions include conditions that are caused by embryologic etiologies as well as those caused by genetic mutations. Patients with genetic etiologies for their craniofacial condition were included in both genetic and craniofacial analyses.

Of the 16 RRT patients who had an identifiable risk factor, 11 (68.8\%) were intubated on the first attempt. However, 2 of 16 (12.5\%) were intubated on the fifth attempt. One of these patients had blood in their airway and the second had a craniofacial anomaly. Prior hospitalization and intubation records showed that both of these patients had been intubated previously without difficulty. 
In multivariable logistic regression (Table 3), having a craniofacial abnormality (adjusted OR [aOR], 51.6; 95\% CI: 2.50-1065.1), airway swelling (aOR, 240.1; 95\% CI: 13.6-4237.2), airway trauma (all trauma patients required DART intubation), and older age (1-2 years vs. <1 year: aOR, 43.3 ; $95 \%$ CI: $2.73-684.3$ and $>5$ years vs. <1 year: aOR, 13.1; 95\% CI: 1.85-93.4) significantly increased odds for requiring the DART. Additionally, as the number of risk factors for a difficult airway increased so did the odds of needing the DART for all risk factors ( $\mathrm{P}<0.001)$. However, airway bleeding (95\% CI, 0.50-75.2), musculoskeletal disease (95\% CI: 0.12145.1), and genetic syndromes without craniofacial abnormalities (95\% CI: 0.15-15.9) did not significantly increase the odds of having a difficult airway that required DART management. The odds for undergoing CPR during intubation were lower in patients cared for by the DART than for those cared for by the RRT (aOR, 0.04; 95\% CI: 0.01-0.23).

\section{Known versus unknown difficult airway history}

Of patients who required the DART, 21 (42\%) did not have a history of difficult airway; intubation was initially attempted by the RRT in 12 of these patients. All patients with a known history of a difficult airway had initial intubation attempts by the DART. DART patients without a history of difficult airway, that is, an unanticipated difficult airway, had risk factors similar to those of patients with a difficult airway history. There were no significant differences in genetic abnormalities $(\mathrm{P}=0.16)$, airway bleeding $(\mathrm{P}=0.20)$, or airway trauma $(\mathrm{P}=0.43)$ between patients with and without a history of difficult airway. Patients with a history of difficult airway had more craniofacial $(\mathrm{P}<0.001)$ and musculoskeletal diseases $(\mathrm{P}=0.04)$ and fewer airway swelling symptoms $(\mathrm{P}=0.04)$ than did patients without a history of a difficult airway (Table 4$)$. 


\section{Cureus}

\begin{tabular}{|c|c|c|c|c|}
\hline Comorbidity ${ }^{a}$ & Total & History of difficult airway $(n=29)$ & No history of difficult airway $(n=21)$ & P-value ${ }^{\mathrm{b}}$ \\
\hline Age, $n(\%)$ & & & & 0.06 \\
\hline$<1$ year & 13 & $8(28)$ & $5(24)$ & \\
\hline 1-2 years & 8 & $7(24)$ & $1(5)$ & \\
\hline $2-5$ years & 3 & 0 & $3(14)$ & \\
\hline$>5$ years & 26 & $14(48)$ & $12(57)$ & \\
\hline Aimway bleeding, n (\%) & 10 & $4(14)$ & 6 (29) & 0.20 \\
\hline Airway swelling, n (\%) & 16 & $6(21)$ & $10(48)$ & 0.04 \\
\hline Trauma, n (\%) & 16 & $8(28)$ & $8(42)$ & 0.43 \\
\hline Genetic syndromes, $n$ (\%) & 4 & 1(4) & $3(14)$ & 0.16 \\
\hline Craniofacial diseases, $n(\%)^{\mathrm{c}}$ & 8 & $13(45)$ & 0 & $<0.001$ \\
\hline Musculoskeletal diseases, $\mathbf{n}(\%)$ & 9 & $8(28)$ & $1(5)$ & 0.04 \\
\hline \multicolumn{5}{|l|}{ Multiple risk factors, } \\
\hline No risk factors & 8 & $6(21)$ & $2(10)$ & 0.29 \\
\hline One risk factor & 21 & $9^{9(31)}$ & $12(57)$ & \\
\hline Two risk factors & 17 & $11(34)$ & $6(29)$ & \\
\hline More than two risk factors & 4 & $3(10)$ & $1(5)$ & \\
\hline
\end{tabular}

\section{TABLE 4: Comparison of patients with known history of difficult airway to patients without a history of difficult airway intubated by the difficult airway response team.}

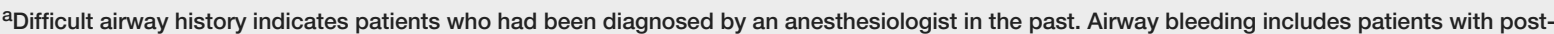
tonsillectomy bleeding and airway trauma. Airway swelling includes patients with anaphylaxis, airway burns or inhalational injury, subglottic stenosis, and airway trauma. Trauma includes patients who had been in a motor vehicle crash, had attempted suicide, or had a gunshot wound. Genetic syndromes include Algille's, Epidermolysis bullosa, and Moya Moya disease. Craniofacial diseases include Treacher-Collins, Pierre-Robin, Noonan's, and Hunter's syndrome. Musculoskeletal diseases include muscular dystrophy.

bStatistical differences were assessed with univariable logistic regression modeling.

${ }^{c}$ Craniofacial conditions include conditions that are caused by embryologic etiologies as well as those caused by genetic mutations. Patients with genetic etiologies for their craniofacial condition were included in Craniofacial analyses only. All patients with a craniofacial abnormality had a history of a difficult airway and none were newly diagnosed.

\section{Discussion}

In this analysis of our institution's RRT and DART databases, we identified several risk factors for difficult airway management that necessitated the DART for airway securement. Risk factors included airway swelling, craniofacial anomalies, trauma, and ages $1-2$ years and $>5$ years. The odds increased for patients who had multiple risk factors. In addition, patients with and without a history of difficulty during airway management had similar risk factors. However, congenital abnormalities were more common in those with difficult airway history. Our findings support the hypothesis that difficult airway management can be predicted in children outside the perioperative setting and led to the creation and deployment of a pediatricspecific DART and consultation service. We believe that this is the first comparison of emergency response teams to evaluate patient comorbidities and scenarios that could predict the need for a hospital-based DART with specialized personnel, equipment, and techniques.

Interestingly, genetic syndromes without craniofacial involvement did not increase the risk for DART intervention, nor did airway bleeding or musculoskeletal diseases. Similar to results published by Sterrett et al. [9], we found that intubation by both the DART and RRT occurred throughout all hours of the day and night. Not only must hospitals have RRTs staffed with expert personnel, these teams must also be readily available 24 hours a day. These findings underline an opportunity for clinicians to consult and create airway management plans for children at risk for a difficult airway prior to respiratory distress and address gaps in staffing, as advanced-level practitioners may be needed during the night. 
Patients with craniofacial abnormalities or airway swelling, or who were 1-2 years old, were significantly more likely to have airway management by the DART in our study than were children without these risk factors. These findings are consistent with previously identified risk factors for difficult airway management in children $[3,9,10]$. Unexpectedly, active chest compressions were more common during intubations performed by the RRT, suggesting that glottic movement did not contribute to the ease of endotracheal tube placement. Patients with airway bleeding did not have an increased requirement for the DART; however, bleeding was not quantified and was likely minimal in these patients. In contrast to literature identifying age less than one year as a risk factor for difficult intubation by anesthesia providers in the OR [2,11,12], our study showed that this age group did not need the DART more frequently than other age groups. This finding is more consistent with results from Graciano et al. [3], who showed in univariate analysis that age $<2$ years was associated with difficult intubation. Unlike previously published literature, we are evaluating difficulty in emergent intubations encountered across multiple subspecialties, including neonatology, pediatric critical care, and emergency medicine. Intensivists from both the NICU and PICU routinely intubate children in this age group, suggesting that age alone does not increase the risk for difficulty during emergent intubation.

Limiting intubation attempts in patients with a history of a difficult airway may benefit significantly from early identification and airway management planning. In our study, patients with a history of difficult airway still required a median of 2.5 attempts by the DART to secure the airway. Performing more than three direct laryngoscopic intubation attempts significantly increases the risk of complications, including hypoxia and cardiac arrest [12]; therefore, efforts to optimize first-attempt success is paramount.

Recognizing the need to keep laryngoscopic attempts to a minimum, in 2015, our institution transitioned to having pediatric anesthesiology attendings in-house 24 hours a day, seven days per week to respond to all pediatric DART calls. In 2017, the pediatric difficult airway consult service was also developed and implemented $[13,14]$. Airway management may not be a priority for non-anesthesiology-trained physicians admitting children without respiratory distress to the hospital, suggesting that automated screening for patients at risk for a difficult airway may be beneficial. Anesthesiologists, pediatric intensivists, neonatologists, and emergency physicians may appreciate early identification of these patients because it would allow time to implement airway management plans and alert the DART prior to respiratory distress.

Certain clinical scenarios can predict difficult airway management. In our study, we found that patients with traumatic injuries who presented to the ED had an increased likelihood of needing the DART. Because prehospital personnel alerts the ED before arrival, early identification of airway trauma can be communicated. Thus, the DART could be activated before patient arrival. Specifically, clinicians must execute extensive preparation prior to conducting airway management for patients under investigation for SARS-CoV-2 infection, even if they do not have a difficult airway history [15]. Thus, early screening and implementation of airway management plans for patients under investigation for difficult airway could optimize firstattempt success and minimize advanced airway equipment contamination and clinician exposure during a respiratory emergency.

The most common locations for pediatric intubations by both the RRT and DART were the ICUs and ED. Advanced airway equipment was also needed more frequently for DART intubations than for RRT intubations. DART carts stocked with advanced airway equipment [5] may be strategically placed throughout the hospital in higher-volume locations. The database records showed that physicians commonly used operative laryngoscopy with rigid bronchoscopes, flexible bronchoscopes, and videolaryngoscopes for emergency airway management. Therefore, personnel on the DART should be regularly trained in the use of this equipment [16]. Creating, maintaining, and processing the equipment on each cart is time-consuming and costly, and having carts at each high-volume location may not be financially feasible for all institutions. Development of one mobile cart may be more realistic for lower-volume facilities.

\section{Limitations}

There are several limitations to this study. First, because this was a retrospective analysis of a prospective database, some real-time data associated with each intubation were missing. Specifically, the rank of the physician who successfully placed the endotracheal tube was present only half of the time. Second, in some situations, the DART should have been activated but was not. One patient underwent six intubation attempts by the RRT, a number significantly greater than the maximum of three attempts that policy recommends before initiating the DART. Third, many critical care physicians who respond during RRT calls at our institution have dual training in both anesthesiology and pediatric critical care. These physicians have advanced training in airway management and may not require backup assistance from the DART; however, if many anesthesia-trained intensivists were intubating as members of the RRT, our results would have shown fewer differences between the RRT and DART groups. This added level of expertise may reduce the generalizability of our single-center study. Fourth, active CPR was performed more commonly during RRT intubation; however, whether compressions were immediately halted during endotracheal tube placement is unknown. Lastly, patients with a difficult airway history all received a DART page at the onset of respiratory distress. This practice precluded the RRT from making any initial attempts at intubation and potentially biases the results for predicting the need for the DART over the RRT in this patient group. To address this bias, we performed a comparison between patients with and without a history of difficult airway. 


\section{Conclusions}

Respiratory compromise in children can occur day or night, but patients with a difficult airway can often be predicted. Having a designated difficult airway management team in the hospital that is always available and knowledgeable in advanced airway techniques is a central component of optimizing safety for pediatric inpatients. Identifying at-risk patients and implementing airway management plans prior to respiratory distress may improve first-attempt intubation success.

\section{Additional Information \\ Disclosures}

Human subjects: Consent was obtained or waived by all participants in this study. Johns Hopkins IRB issued approval NA_00089582. Animal subjects: All authors have confirmed that this study did not involve animal subjects or tissue. Conflicts of interest: In compliance with the ICMJE uniform disclosure form, all authors declare the following: Payment/services info: All authors have declared that no financial support was received from any organization for the submitted work. Financial relationships: All authors have declared that they have no financial relationships at present or within the previous three years with any organizations that might have an interest in the submitted work. Other relationships: All authors have declared that there are no other relationships or activities that could appear to have influenced the submitted work.

\section{References}

1. Jagannathan N, Kho MF, Kozlowski RJ, Sohn LE, Siddiqui A, Wong DT: Retrospective audit of the air-Q intubating laryngeal airway as a conduit for tracheal intubation in pediatric patients with a difficult airway. Paediatr Anaesth. 2011, 21:422-7. 10.1111/j.1460-9592.2010.03494.x

2. Heinrich S, Birkholz T, Ihmsen H, Irouschek A, Ackermann A, Schmidt J: Incidence and predictors of difficult laryngoscopy in 11,219 pediatric anesthesia procedures. Paediatr Anaesth. 2012, 22:729-36. 10.1111/j.1460-9592.2012.03813.x

3. Graciano AL, Tamburro R, Thompson AE, Fiadjoe J, Nadkarni VM, Nishisaki A: Incidence and associated factors of difficult tracheal intubations in pediatric ICUs: a report from National Emergency Airway Registry for Children: NEAR4KIDS. Intensive Care Med. 2014, 40:1659-69. 10.1007/s00134-014-3407-4

4. Gosfield AG, Reinertsen JL: The 100,000 lives campaign: crystallizing standards of care for hospitals . Health Aff. 2005, 24:1560-70. 10.1377/hlthaff.24.6.1560

5. Mark LJ, Herzer KR, Cover R, et al.: Difficult airway response team: a novel quality improvement program for managing hospital-wide airway emergencies. Anesth Analg. 2015, 121:127-39. 10.1213/ANE.0000000000000691

6. Russo SG, Becke K: Expected difficult airway in children . Curr Opin Anaesthesiol. 2015, 28:321-6. 10.1097/ACO.0000000000000198

7. Daigle CH, Fiadjoe JE, Laverriere EK, et al.: Difficult bag-mask ventilation in critically ill children is independently associated with adverse events. Crit Care Med. 2020, 48:e744-52. 10.1097/CCM.0000000000004425

8. Duval-Arnould JM, Newton HM, McNamara L, et al.: Design and deployment of a pediatric cardiac arrest surveillance system. Crit Care Res Pract. 2018, 2018:9187962. 10.1155/2018/9187962

9. Sterrett EC, Myer CM 4th, Oehler J, Das B, Kerrey BT: Critical airway team: a retrospective study of an airway response system in a pediatric hospital. Otolaryngol Head Neck Surg. 2017, 157:1060-7. 10.1177/0194599817719400

10. Sims C, von Ungern-Sternberg BS: The normal and the challenging pediatric airway. Paediatr Anaesth. 2012, 22:521-6. 10.1111/j.1460-9592.2012.03858.x

11. Gálvez JA, Acquah S, Ahumada L, et al.: Hypoxemia, bradycardia, and multiple laryngoscopy attempts during anesthetic induction in infants: a single-center, retrospective study. Anesthesiology. 2019, 131:8309. 10.1097/ALN.0000000000002847

12. Fiadjoe JE, Nishisaki A, Jagannathan N, et al.: Airway management complications in children with difficult tracheal intubation from the Pediatric Difficult Intubation (PeDI) registry: a prospective cohort analysis. Lancet Respir Med. 2016, 4:37-48. 10.1016/S2213-2600(15)00508-1

13. Dalesio NM, Diaz-Rodriguez N, Koka R, et al.: Development of a multidisciplinary pediatric airway program: an institutional experience. Hosp Pediatr. 2019, 9:468-75. 10.1542/hpeds.2018-0226

14. Nykiel-Bailey SM, McAllister JD, Schrock CR, Molter DW, Marsh JK, Murray DJ: Difficult airway consultation service for children: steps to implement and preliminary results. Paediatr Anaesth. 2015, 25:363-71. 10.1111/pan.12625

15. Matava CT, Kovatsis PG, Lee JK, et al.: Pediatric airway management in COVID-19 patients: consensus guidelines from the Society for Pediatric Anesthesia's Pediatric Difficult Intubation Collaborative and the Canadian Pediatric Anesthesia Society. Anesth Analg. 2020, 131:61-73. 10.1213/ANE.0000000000004872

16. Leeper WR, Haut ER, Pandian V, et al.: Multidisciplinary difficult airway course: an essential educational component of a hospital-wide difficult airway response program. J Surg Educ. 2018, 75:1264-75. 10.1016/j.jsurg.2018.03.001 